\title{
Combate ao Cancro da Mama Invasivo com Fármacos de Ruténio inmenconateat
}

\author{
Fighting Triple-Negative Breast Cancer with \\ Ruthenium-based Metallodrugs. Breast \\ cancer is the most common type of cancer \\ and the second leading cause of death in \\ women worldwide. In particular, triple- \\ negative breast cancer (TNBC) has a very \\ poor prognosis, and no effective treatment is \\ currently available. The group of Bioinorganic \\ Chemistry and Drug Development (BIOIN) \\ from Faculdade de Ciências da ULisboa is \\ exploring two different approaches to target \\ TNBC: passive and active targeting, by \\ using macromolecules and/or biomolecules, \\ respectively, in the design of new compounds \\ for selectivity enhancement. Recent results \\ regarding two promising compounds with \\ the general formula $\left[\mathrm{Ru}(\mathrm{Cp})\left(\mathrm{PPh}_{3}\right)(\mathrm{bpy}-\mathrm{R})\right]^{+}$, \\ bpy $=2,2$ '-bipyridine and $R=$ biotin (LCR134) \\ or a polylactide (PMC78), disclosed promising \\ biological activity. Overall, these results \\ prove that the present new compounds \\ bring innovation on the design of potential \\ drugs for chemotherapy due to their anti- \\ metastatic potential, ability to overcome \\ multidrug resistance and reduced toxicity \\ (zebrafish model).
}

\begin{abstract}
O cancro da mama é o tipo de cancro mais comum e a segunda causa de morte nas mulheres a nível mundial. Em particular, o cancro da mama triplo-negativo (TNBC) tem um mau prognóstico uma vez que não possui qualquer tratamento eficaz. O grupo Bioinorganic Chemistry and Drug Development (BIOIN) da Faculdade de Ciências da ULisboa tem explorado estratégias de síntese de novos compostos organometálicos de ruténio baseadas no conceito de "tiro ao alvo", i.e., direcionamento e acumulação específica no tumor, através de fenómenos de direcionamento ativo e passivo. O sucesso destas estratégias está refletido nos resultados obtidos para dois compostos da família $\left[R u(C p)\left(P P h_{3}\right)(b p y-R)\right]^{+}, b p y=2,2^{\prime}-b i^{-}$ piridina e $R$ = biotina (LCR134) ou ácido poliláctico (PMC78) que revelaram uma atividade biológica promissora nas células TNBC, muito agressivas e metastáticas. Mostraram ainda capacidade para ultrapassar mecanismos de resistência, potencial antimetastático e baixa toxicidade (modelo do peixe zebra). O sucesso da estratégia inovadora aqui apresentada constitui uma abordagem promissora a ser aplicada no design de potenciais novos fármacos.
\end{abstract}

\section{Introdução \\ 1.1. Cancro da mama}

Segundo os dados da Organização Mundial de Saúde, o cancro da mama é o tumor maligno com maior incidência e também a principal causa de morte em mulheres a nível mundial. Estima-se que em 2020 este cancro será responsável por cerca de 650 mil mortes de um total de 2,2 milhões de casos diagnosticados [1]. Dos vários subtipos de cancro da mama conhecidos, o cancro da mama triplo-negativo é caracterizado pela ausência da expressão de recetores hormonais, nomeadamente estrogénio e progesterona, e ausência da expressão do recetor tipo 2 do fator de crescimento epidérmico humano (HER2), que tem um papel fundamental no crescimento e desenvolvimento de uma vasta categoria de células, designadas por células epiteliais [2]. Este subtipo de cancro da mama é conhecido por ser biologicamente mais agressivo e não ter um alvo terapêutico identificado, pelo que a procura de fármacos alternativos e eficazes para o seu tratamento é crucial. 


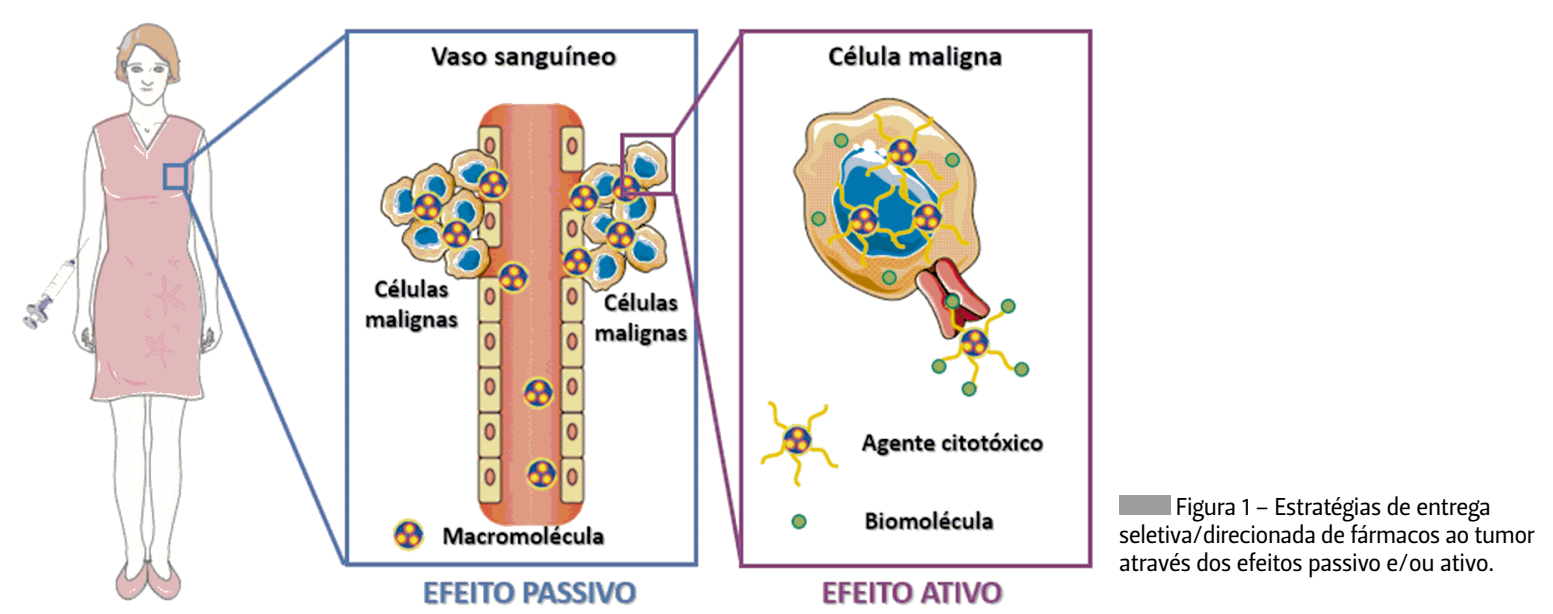

\subsection{Estratégias de tratamento:}

\section{direcionamento passivo e ativo}

Um dos principais desafios da quimioterapia consiste na descoberta de novos fármacos capazes de atingir o(s) local(is) do tumor, deixando intactas as células saudáveis, evitando assim os efeitos secundários. Para alcançar esse objetivo, estão atualmente em aplicação ou em desenvolvimento vários sistemas de administração de fármacos tais como micelas [3-6], lipossomas [7-10], conjugados metal-polímero [11,12] ou dendrímeros $[13,14]$. Existem duas estratégias amplamente utilizadas para a entrega de fármacos ao órgão/tecido desejado: o direcionamento passivo e o direcionamento ativo (Figura 1).

A estratégia de direcionamento passivo é baseada num fenómeno designado por efeito de permeabilidade e retenção aumentada (EPR, do inglês Enhanced Permeability and Retention Effect), descrito pela primeira vez por Maeda et al. em 1986 [15], o qual ocorre na grande maioria dos tumores sólidos humanos. Este conceito baseia-se no facto das células tumorais, na ausência de oxigénio (hipóxia), terem um crescimento acelerado relativamente às células saudáveis, ativando fatores de crescimento (VEGF - fator de crescimento vascular endotelial) para a produção de novos vasos sanguíneos, processo conhecido por angiogénese, com vista à obtenção de oxigénio e nutrientes. Contudo, esta hipervascularização anormal produz vasos defeituosos e de elevada permeabilidade, exibindo poros junto do tecido tumoral. Devido a estas características, as macromoléculas tendem a extravasar para o tecido tumoral através desses poros, onde ficam acumuladas em consequência de uma excreção (drenagem linfática) ineficiente. Assim, as macromoléculas podem ser introduzidas no design de novos fármacos, como por exemplo os conjugados metal-polímero, para beneficiar deste efeito. É importante mencionar que os vasos formados durante a angiogénese não são distribuídos uniformemente num tumor sólido e, consequentemente, a permeabilidade pode não ser homogénea, o que faz com que a eficácia do direcionamento passivo possa, por vezes, ser limitada. Assim, outra estratégia que pode ser usada para superar este problema é o direcionamento ativo, pois é uma estratégia complementar bastante promissora para aumentar ainda mais a eficiência dos sistemas de administração de fármacos [16]. O principal mecanismo do direcionamento ativo baseia-se no reconhecimento de uma molécula biologicamente ativa (biomolécula), como por exemplo, anticorpos, péptidos, açúcares, vitaminas, etc., pelo seu substrato/recetor-alvo específico e consequente entrada na célula por endocitose. Esta estratégia baseia-se na sobreexpressão de recetores destas biomoléculas nas células tumorais, comparativamente às células saudáveis [16]. É, então, possível incluir estas duas estratégias promissoras para o direcionamento específico dos fármacos para o tumor (aumento de seletividade).

\subsection{O ruténio como metal promissor no tratamento do cancro}

A descoberta acidental das propriedades antitumorais da cisplatina em 1965 [17] foi um marco na história da quimioterapia onde, pela primeira vez, um metalofármaco entrou em uso clínico para o tratamento do cancro. Apesar da sua inegável importância, a severidade dos seus efeitos secundários limita a dose de tratamento administrada aos pacientes, que pode ser subletal para os tumores, 
levando ao desenvolvimento de resistência das células tumorais com os ciclos de quimioterapia (resistência adquirida), embora alguns tipos de cancro sejam intrinsecamente insensíveis ao tratamento com a cisplatina (resistência intrínseca). Os seus principais efeitos secundários incluem neurotoxicidade (danos no sistema nervoso), nefrotoxicidade (danos na função renal), ototoxicidade (perda auditiva) e mielossupressão (diminuição da atividade da medula óssea). Neste contexto, têm surgido outros metais como possíveis alternativas à platina, como por exemplo o ruténio, que tem demonstrado possuir propriedades interessantes para o desenvolvimento de novos fármacos, tais como baixa toxicidade e ter uma gama de estados de oxidação extensa, e.g. Ru(II), Ru(III) e Ru(IV), acessíveis em condições fisiológicas.

Nas últimas décadas, vários compostos de ruténio foram sintetizados e testados quanto à sua potencial atividade antitumoral. Destes, destacam-se dois compostos de Ru(III), NAMI-A (trans-[tetracloro(1H-imidazol)(dimetilssulfóxido)rutenato(III)] de imidazólio) desenvolvido por Sava et al. [18] e KP1019 (trans-[tetraclorobis(1H-indazol)rutenato(III)] de indazólio), desenvolvido por Keppler et al. [19] que, apesar dos resultados promissores (potencial antimetastático e atividade para o tumor principal, respetivamente), acabaram por ser excluídos dos ensaios clínicos devido a problemas de formulação. Mais tarde, este problema foi contornado pela síntese do sal de sódio análogo ao KP1019, isto é, o composto NKP-1339 (trans-[tetraclorobis $(1 \mathrm{H}$-indazol)rutenato(III)]) de sódio) [20] que é 35 vezes mais solúvel do que o seu análogo. Este composto já completou a fase I dos ensaios clínicos, no que respeita à sua segurança, tolerabilidade, farmacocinética e farmacodinâmica e os resultados deste estudo apontam este fármaco como um candidato promissor para estudos ainda da fase I, mas agora em combinação com outros fármacos e estudos da fase II que estão a ser planeados [21,22].

Foram também desenvolvidos por Sadler et al. e Dyson et al. complexos organometálicos de ruténio(II)- $\left(\eta^{6}-\mathrm{C}_{6} \mathrm{H}_{6}\right)$ com estrutura em banco de piano. Estes compostos mostraram boa atividade in vitro e in vivo [23] apresentando, em alguns casos, atividade antimetastática [24], abrindo caminho para o desenvolvimento de novos agentes organometálicos de ruténio-areno.

\subsection{Complexos de ruténio- ciclopentadienilo (RuCp)}

Durante a última década, o grupo Bioinorganic Chemistry and Drug Development (BIOIN) do CQE - Ciências, Centro de Química Estrutural, tem desenvolvido uma ampla família de compostos baseados no fragmento ruténio-ciclopentadienilo, "Ru $\left(\eta^{5}-\mathrm{C}_{5} \mathrm{H}_{5}\right)$ ", com vista a uma potencial aplicação futura na terapia do cancro. A maioria destes compostos organometálicos tem a fórmula geral $[\mathrm{Ru}(\mathrm{CP})$ $\left.(\mathrm{L})(\mathrm{P})_{n}\right][\mathrm{X}](\mathrm{P}=$ fosfano; $\mathrm{L}=$ ligando heteroaromático; $\mathrm{X}=\mathrm{CF}_{3} \mathrm{SO}_{3}^{-}$ou $\mathrm{PF}_{6}^{-} ; \mathrm{n}=1$ ou 2 ) e uma geometria em banco de piano, em que três posições de coordenação são ocupadas pelo anel ciclopentadienilo, permitindo a introdução de ligandos heteroaromáticos (mono ou bidentados) e/ou ligandos fosfano (mono ou bidentados) nas três restantes posições. Um dos compostos desta família, TM34, com a fórmula geral $\left[\mathrm{Ru}(\mathrm{Cp})\left(\mathrm{PPh}_{3}\right)(\mathrm{bpy})\right]^{+}$apresentou uma atividade biológica bastante promissora (na gama sub-micromolar) contra um amplo painel de linhas celulares cancerígenas, incluindo a linha celular do carcinoma do ovário resistente à cisplatina (A2780CisR), o adenocarcinoma da mama agressivo e metastático (MDA-MB-231), o carcinoma da próstata grau IV (PC3) e numa linha celular de leucemia humana (HL60) [25]. Os resultados excecionais do TM34 levaram à sua seleção para estudos pré-clínicos num modelo de ratinho (nude mouse model). Contudo, este composto mostrou possuir um perfil tóxico neste modelo animal [26]. No entanto, a estabilidade das suas soluções, comprovada por ultravioleta-visível (UV-Vis) e Ressonância Magnética Nuclear (RMN) por um período de $30 \mathrm{~h}$ em solventes coordenantes (ex. DMSO) e em meio aquoso tamponado [25], demonstraram que este pode constituir um bom modelo e ser incluído nas estratégias de design dos novos fármacos com vista ao direcionamento (ativo e/ou passivo) para as células tumorais.

\section{Resultados e discussão}

De forma a suprimir a aparente falta de seletividade do composto TM34, criou-se uma nova família de compostos cujo racional de síntese foi a conjugação do fragmento citotóxico presente na estrutura do TM34, ou seja "Ru(Cp)(PPh $)$ (bpy)", de elevada estabilidade, a uma biomolécula (biotina, LCR134) [27] ou a um macroligando (ácido poliláctico, PMC78) [12], para beneficiar dos efeitos de direcionamento ativo ou passivo, respetivamente, para as células tumorais. Os compostos LCR134, PMC78 e o composto análogo sem biomolécula ou macroligando PMC79 (Figura 2) foram testados em diversos ensaios celulares in vitro em duas linhas distintas do cancro da mama, MCF7 (hormono-dependente) e MDA-MB-231 (modelo do cancro da mama triplo-negativo), ambas apresentando sobreexpressão de recetores de biotina (SMVT) $[12,27]$. Foi ainda avaliada a toxicidade preliminar de dois destes compostos no modelo do peixe-zebra [27]. 


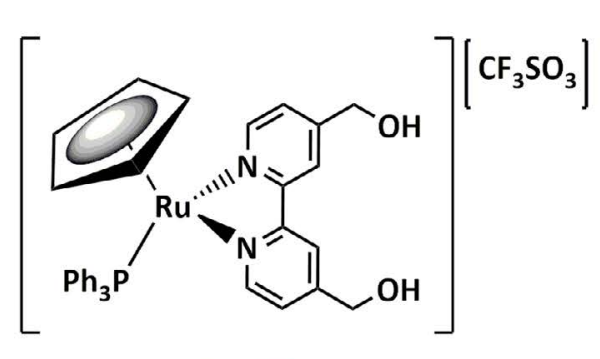

PMC79

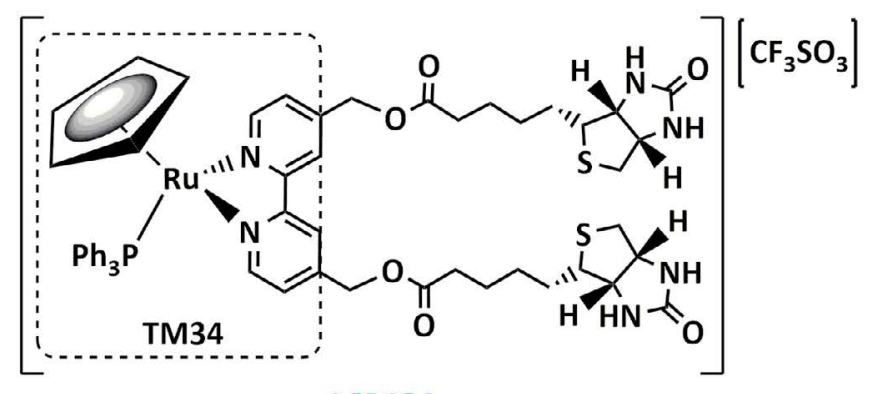

LCR134

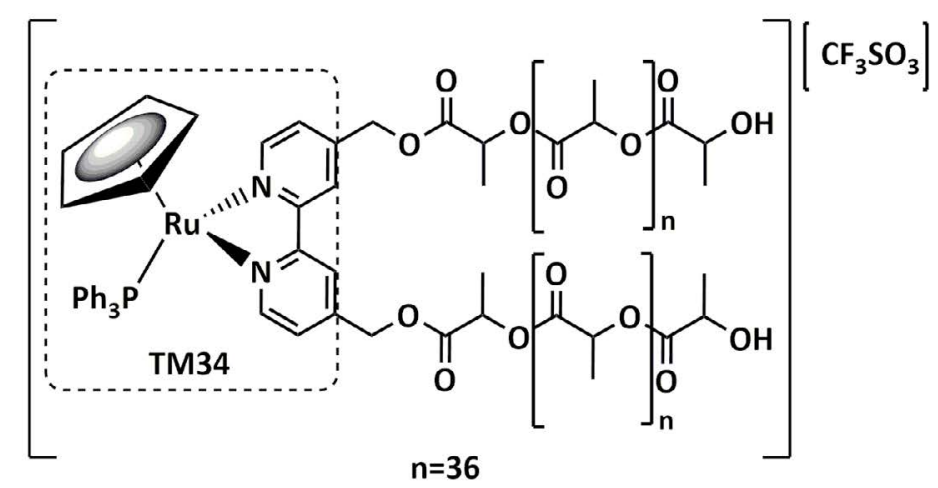

PMC78
Figura 2 - Estrutura química dos complexos de ruténiociclopentadienilo: PMC79 - sem biomolécula ou macroligando, LCR134 - com biomolécula (biotina) e PMC78 com macroligando de ácido poliláctico.

\subsection{Atividade biológica e tipo de morte celular} Os resultados preliminares de atividade biológica dos compostos PMC79, LCR134 e PMC78 demonstraram que todos são bastante mais citotóxicos do que a cisplatina (fármaco de referência). 0 composto PMC78, contendo a macromolécula com vista ao direcionamento passivo, foi o que obteve um valor de $I_{50}$ (concentração capaz de inibir $50 \%$ das células) mais baixo em ambas as linhas celulares e em particular na linha mais agressiva (MDA-MB-231) - Figura 3. De facto, é interessante observar que nos compostos com vista ao direcionamento ativo (LCR134) e passivo (PMC78), se verificou um aumento da atividade biológica na linha mais agressiva, comparativamente à linha hormono-dependente (MCF7), o que poderá estar relacionado com diferenças genéticas entre estas duas linhas [12,27].

Quanto ao tipo de morte celular, existem dois tipos principais: apoptose e necrose. A apoptose é uma forma de morte celular programada ou "suicídio celular" de extrema importância para a renovação dos tecidos, promovendo o equilíbrio do organismo, enquanto que a necrose é um processo desorganizado que origina quase sempre uma resposta inflamatória, pelo que a apoptose é geralmente aceite como o mecanismo preferencial de morte celular. Neste contexto, foi avaliado o tipo de morte celular dos compostos PMC79, LCR134 e PMC78 na concentração equivalente ao $\mathrm{IC}_{50}$ e ao dobro do $\mathrm{IC}_{50}$ nas células do cancro da mama triplo negativo (MDA-MB-231), utilizando a técnica de citometria de fluxo, verificando-se que todos potenciam uma morte celular por apoptose $[12,28]$.

Figura 3 - Valores de IC ${ }_{50}$ dos compostos PMC79, LCR134, PMC78 e cisplatina (CDDP) nas linhas celulares do carcinoma da mama, MCF7 (hormono-dependente) e MDA-MB-231 (modelo do cancro da mama triplonegativo), às $24 \mathrm{~h}$ de incubação.

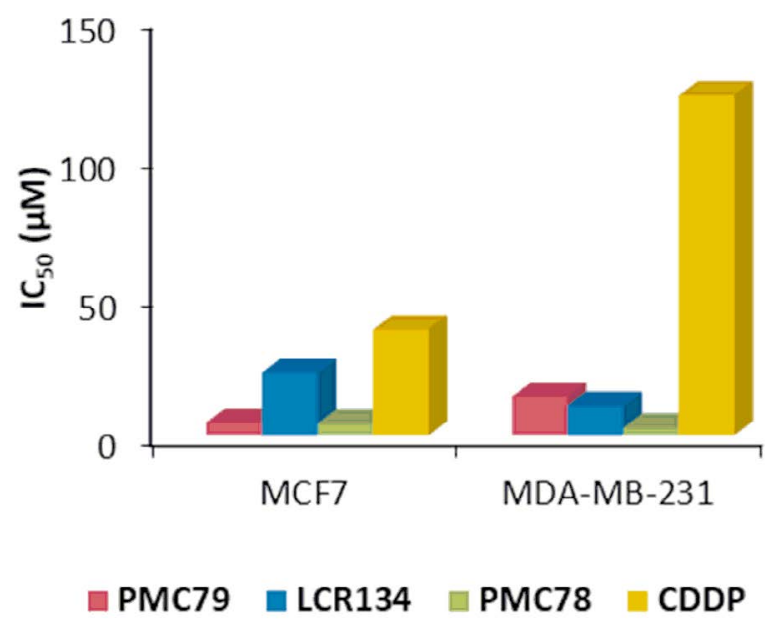




\subsection{Ensaio de interação biotina-avidina}

Para averiguar se a ligação da biotina ao complexo de ruténio poderia comprometer o reconhecimento do complexo final (LCR134) pelos recetores/ transportadores da biotina (SMVT) foi realizado um ensaio in vitro geralmente utilizado para fazer uma avaliação preliminar desse reconhecimento, através da interação entre a biotina e a proteína avidina, pois a afinidade relativa entre estes é elevada. Este ensaio permitiu verificar que a ligaçãa da biotina ao complexo não afetou o reconhecimento do complexo final pela avidina, validando assim a nossa estratégia inicial que consiste em promover a entrada para as células tumorais através dos recetores de biotina (direcionamento ativo) [28].

\subsection{Ensaio de inibição da formação de colónias}

Foi igualmente averiguado o potencial de inibição da formação de colónias na linha mais agressiva (MDA-MB-231) dos compostos PMC79, LCR134 e PMC78. Este ensaio permite determinar a capacidade que as células possuem de sobreviver à exposição a um agente quimioterapêutico por um curto período e posterior capacidade de sobreviver, isto é, de produzir colónias após a remoção desse agente, simulando in vitro o que acontece durante os ciclos de quimioterapia. Os resultados deste ensaio mostraram que todos os compostos têm a capacidade de inibir a formação de colónias nesta linha, demonstrando ter uma potencial capacidade antimetastática $[12,28]$.

\subsection{Distribuição celular e estudos de proteómica para avaliação dos potenciais alvos celulares}

De forma a inferir acerca da possível localização dos alvos celulares destes compostos, foi realizado um ensaio de distribuição celular e posterior quantificação dos níveis de ruténio nos seus principais compartimentos (citoesqueleto, citosol, membrana e núcleo). Assim, as células do cancro da mama mais agressivo (MDA-MB-231) foram incubadas com os compostos PMC79, LCR134 e PMC78 na concentração equivalente ao seu IC ${ }_{50}$, durante $24 \mathrm{~h}$. Seguidamente, foi utilizado um kit de fracionamento para fragmentar a célula nos seus compartimentos principais e os níveis de ruténio nestes organelos foram quantificados através da técnica de Espectrometria de Massa acoplada a plasma indutivo, ICP-MS $[12,28]$. Os resultados de quantificação do ruténio indicam que os compostos PMC79 e LCR134 se acumulam preferencialmente na membrana celular (Figura 4), o que poderá indicar que têm como possíveis alvos proteínas membranares. Por outro lado, o PMC78 acumula-se preferencialmente no citoesqueleto das células [12,28] (Figura 4).
Figura 4 - Quantificação do ruténio nos principais organelos (citosol, membrana, núcleo e citoesqueleto) das células MDAMB-231 tratadas com os compostos PMC79, LCR134 e PMC78, na concentração equivalente ao $\mathrm{IC}_{50}$, às $24 \mathrm{~h}$ de incubação.

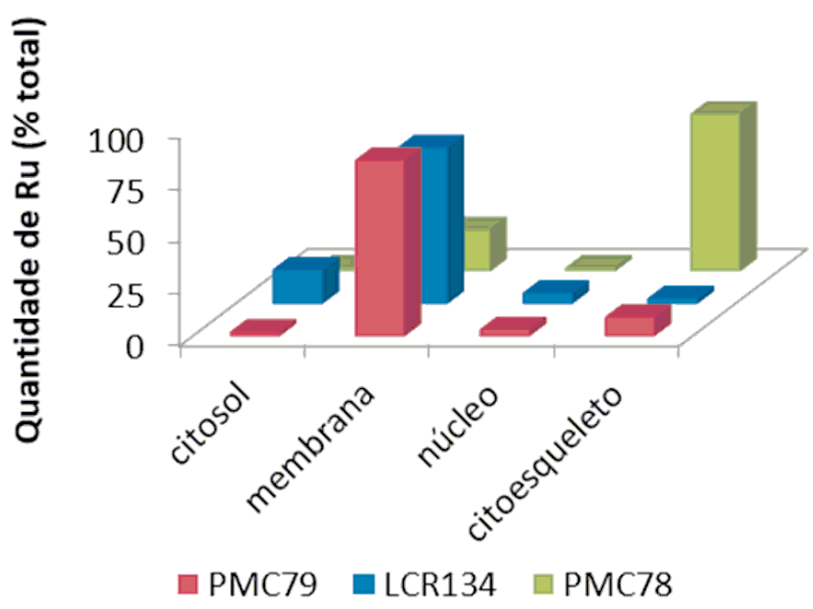

Foram ainda realizados estudos de proteómica na linha celular MDA-MB-231 para identificar possíveis alterações na expressão de diferentes proteínas. Verificou-se que o alvo principal do composto PMC79 são as proteínas que regulam a dinâmica da actina, responsável pela contração celular, atividades de endocitose e exocitose, e migração celular. No caso do PMC78, o principal alvo são as proteínas que regulam a dinâmica dos microtúbulos, que desempenham um papel fundamental na organização e no suporte estrutural da célula [12]. Quanto ao composto LCR134, embora não tenham sido realizados estudos de proteómica, resultados de imunofluorescência mostraram que este composto, tal como o seu análogo sem a biomolécula (PMC79), induz alterações na organização da actina (resultados não publicados). No geral, estes resultados são bastante interessantes pois enquadram estes compostos numa recente categoria de agentes denominados migrastatics, ou seja, que têm como possíveis alvos mecanismos essenciais (mobilidade, organização e suporte celular), interferindo com os modos de invasão das células cancerígenas e, consequentemente, com a sua capacidade de metastizar, o que é particularmente importante no tratamento dos cancros mais agressivos e metastáticos [29].

\subsection{Efeito dos complexos na multirresistência} A multirresistência é o principal mecanismo pelo qual muitos cancros desenvolvem resistência à quimioterapia ao longo do tempo, através da expulsão (efluxo), por proteínas de transporte, dos agentes quimioterapêuticos para fora da célula e/ou diminuição da sua internalização. Estas proteínas de transporte, como são exemplo os transportadores $A B C$, encontram-se sobreexpressas nas células tumorais. Neste contexto, foi realizado um estudo que pretendia, por um lado 
avaliar se as proteínas envolvidas na multirresistência (P-gp, MRP1, MRP2 e BCRP), pertencentes à família dos transportadores $A B C$, eram capazes de promover o efluxo dos compostos de Ru (PMC79 e LCR134) para fora da célula e, por outro lado, investigar se estes compostos poderiam atuar como inibidores de algum destes transportadores. Enquanto que o PMC79 não mostrou qualquer inibição dos transportadores ABC, o LCR134 demonstrou possuir uma notável inibição seletiva da proteína P-gp, bem como uma atividade biológica promissora na linha celular mais agressiva do cancro da mama, ou seja, possui um raro e importante efeito dual. Até à data, este é o único composto organometálico de ruténio alguma vez reportado na literatura que atua como agente citotóxico e, em simultâneo, como inibidor de uma proteína envolvida na multirresistência ao cancro, mostrando a relevância das estratégias adotadas para a criação desta nova família de compostos [27].

\subsection{Avaliação da toxicidade usando o modelo de peixe zebra}

0 modelo de peixe zebra tem sido amplamente utilizado na investigação científica, pois possui diversas características que o tornam um modelo valioso para o estudo da genética e das doenças humanas, tais como uma elevada semelhança genética com o ser humano [30]. Este modelo in vivo permite também avaliar a toxicidade de novos fármacos, ajudando a estabelecer prioridades na escolha dos compostos com propriedades biológicas mais interessantes para serem testados em ensaios pré-clínicos. Nesse sentido, os compostos PMC79 (sem biomolécula), LCR134 (com biomolécula, biotina) e análogos ao composto TM34, com a estrutura geral $\left[\mathrm{Ru}\left(\eta^{5}-\mathrm{C}_{5} \mathrm{H}_{5}\right)\left(2,2^{\prime} \text {-bipiridina }\right)\left(\mathrm{P}\left(\mathrm{C}_{6} \mathrm{H}_{4} \mathrm{R}\right)_{3}\right)\right]^{+}$, em que $\mathrm{R}=\mathrm{F}$ ou $\mathrm{OCH}_{3}$, foram testados em peixes zebra para avaliação da sua toxicidade. Verificou-se que os compostos análogos ao TM34, contendo a 2,2'-bipiridina, provocam necrose severa nos peixes após exposição ao composto (Figura 5-A), o que se assemelha aos resultados de toxicidade verificados anteriormente no modelo do ratinho para o TM34. Relativamente ao composto sem a biomolécula, PMC79, apesar de não ter apresentado efeitos de necrose, mostrou causar alguma hemorragia nos peixes e edemas do saco vitelino e do saco pericárdico (Figura 5-B). Pelo contrário, o composto contendo a biomolécula (LCR134) não mostrou efeitos tóxicos muito graves, tendo apenas provocado edema do saco vitelino e do saco pericárdico nas doses mais altas (Figura 5-C) [27,28,31]. Estes resultados preliminares demonstram que a estratégia inicial de síntese dos complexos com vista ao direcionamento ativo foi bem-sucedida, pois o composto contendo a biomolécula foi o que mostrou uma melhor tolerabilidade in vivo e ausência de efeitos tóxicos severos.

\section{Conclusões}

Neste trabalho apresentam-se os resultados promissores para dois novos complexos de ruténio que revelaram um grande potencial para ultrapassar os três principais problemas da quimioterapia: i) falta de seletividade para as células tumorais; ii) metástases e iii) multirresistência. A estratégia para o design e síntese destes compostos teve em vista ultrapassar a falta de seletividade dos fármacos atuais, utilizando o conceito de quimioterapia dirigida: eliminar as células cancerígenas poupando as saudáveis. Assim, as novas moléculas contêm na sua estrutura uma biomolécula com vista ao direcionamento ativo ou uma macromolécula para o direcionamento passivo.

Os estudos in vitro permitiram concluir que estes compostos possuem uma atividade biológica bastante promissora na linha celular mais agressiva do cancro da mama e que estes podem interferir com os modos de invasão das células cancerígenas, de extrema importância quando se pretende desenvolver novos fármacos para a terapia de cancros agressivos e metastáticos, como é o caso do cancro da mama triplo-negativo. Um dos compostos mostrou ainda ser um excelente inibidor da proteína P-gp, envolvida na multirresistência, e estudos preliminares de toxicidade no modelo de peixe zebra revelaram que este possui boa tolerabilidade in vivo e ausência de efeitos tóxicos severos.

Globalmente, estes resultados demonstram que foram desenvolvidos compostos menos tóxicos, que
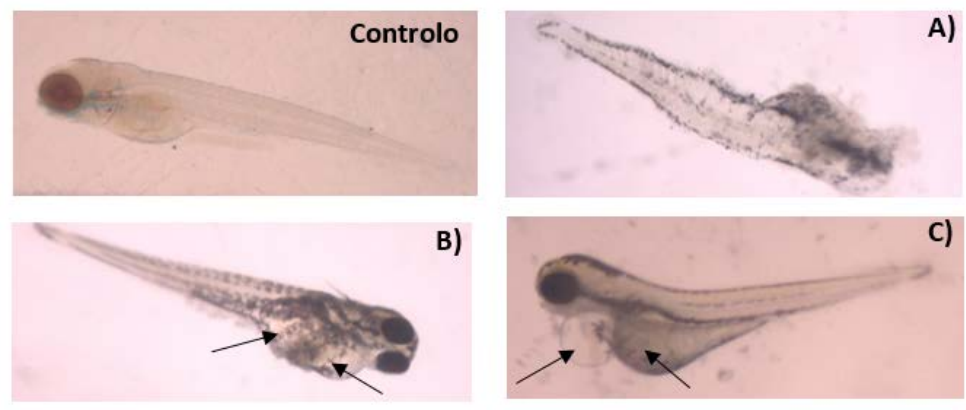

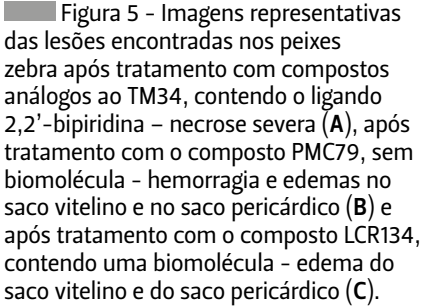


conseguem contornar a falta de seletividade dos fármacos atuais, apresentando potencial antimetastático e capacidade para ultrapassar mecanismos de resistência, o que representa uma enorme conquista uma vez que a principal causa de morte por cancro deve-se às metástases e uma das principais causas de falha na quimioterapia é a multirresistência aos fármacos.

Face aos resultados promissores obtidos neste trabalho, espera-se que estas estratégias de design possam ser aplicadas no futuro à síntese de outros compostos igualmente promissores.

\section{Agradecimentos}

Este trabalho foi financiado pela Fundação Portuguesa para a Ciência e a Tecnologia (FCT) - projetos UID/
QUI/00100/2019 e PTDC/QUI-QIN/28662/2017. A autora, atual Investigadora Júnior no projeto Lead4Target (PTDC/QUI-QIN/28662/2017), agradece à FCT pela concessão da sua bolsa de Doutoramento (SFRH/ BD/100515/2014), à Fulbright pela possibilidade de realização dos estudos in vivo no modelo de peixe zebra na Universidade de Rutgers, E.U.A. e à Comissão Científica do 13. Encontro Nacional de Química Orgânica e 6. Encontro Nacional de Química Terapêutica pelo prémio de melhor comunicação oral. Por último, a autora gostaria de fazer um agradecimento especial às suas orientadoras de doutoramento, Doutora Andreia Valente e Professora Maria Helena Garcia por todo o apoio e ajuda na concretização deste trabalho e à direção do Química, Boletim da SPQ, pelo convite.

\section{Referências}

[1] International Agency for Research on Cancer: gco.iarc.fr/tomorrow/home (acedido em 05/03/2020).

[2] F. Cardoso, "100 perguntas chave no cancro da mama", Permanyer Portugal, Lisboa, 2015.

[3] Y. Li, T. Zhang, Q. Liu, J. He, Front. Pharmacol. 2019, 10, 1-14. DOI: 10.3389/ fphar.2019.00808

[4] G. Yu, Q. Ning, Z. Mo, S. Tang, Artif. Cells Nanomed. Biotechnol. 2019, 47, 1476-1487. DOI: 10.1080/21691401.2019.1601104.

[5] A. Zajdel, A. Wilczok, K. Jelonek, M. Musiat-Kulik, A. Foryś, S. Li, J. Kasperczyk, Pharmaceutics 2019, 11, 169. DOI: 10.3390/pharmaceutics11040169.

[6] S. Zhen, X. Yi, Z. Zhao, X. Lou, F. Xia, B. Z. Tang, Biomaterials 2019, 218, 119330. DOl: 10.1016/j.biomaterials.2019.119330.

[7] E. Beltrán-Gracia, A. López-Camacho, I. Higuera-Ciapara, J. B. VelázquezFernández, A. A. Vallejo-Cardona, Cancer Nanotech. 2019, 10, 11. DOI: 10.1186/s12645-019-0055-y.

[8] C. Hong, D. Wang, J. Liang, Y. Guo, Y. Zhu, J. Xia, J. Qin, H. Zhan, J. Wang, Theranostics 2019, 9, 4437-4449. DOI: 10.7150/thno.34953.

[9] P. Mandpe, B. Prabhakar, P. Shende, Stem Cell Rev. Rep. 2020, 16, 103-117. DOI: 10.1007/s12015-019-09933-z.

[10] B. Tang, Y. Peng, Q. Yue, Y. Pu, R. Li, Y. Zhao, L. Hai, L. Guo, Y. Wu, Eur. J. Med. Chem. 2020, 193, 112204. DOI: 10.1016/j.ejmech.2020.112204.

[11] H. Sun, L. Yan, M. Y. Z. Chang, K. A. Carter, R. Zhang, L. Slyker, J. F. Lovell, Y. Wu, C. Cheng, Nanoscale Adv. 2019, 1, 2761-2771. DOl: 10.1039/C9NA00282K.

[12] T. Moreira, R. Francisco, E. Comsa, S. Duban-Deweer, V. Labas, A. TeixeiraGomes, L. Combes-Soia, F. Marques, A. Matos, A. Favrelle, C. Rousseau, P. Zinck, P. Falson, M. H. Garcia, A. Preto, A. Valente, Eur. J. Med. Chem. 2019 168, 373-384. DOI: 10.1016/j.ejmech.2019.02.061.

[13] S. R. Ambekar, M. Choudhary, B. Kandasubramanian, Eur. Pol. J. 2020, 126, 109546. DOI: 10.1016/j.eurpolymj.2020.109546.

[14] N. T. T. Le, T. N. Q. Nguyen, V. D. Cao, D. T. Hoang, V. C. Ngo, T. T. H. Thi, Pharmaceutics 2019, 11, 110591. DOI: 10.3390/pharmaceutics11110591.

[15] Y. Matsumura, H. A. Maeda, Cancer Res. 1986, 46, 6387-6392. cancerres. aacrjournals.org/content/46/12_Part_1/6387.

[16] M. F. Attia, N. Anton, J. Wallyn, Z. Omran, T. F. Vandamme, J. Pharm. Pharmacol. 2019, 71, 1185-1198. DOI: 10.1111/.jphp.13098.

[17] B. Rosenberg, L. V. Camp, T. Krigas, Nature 1965, 205, 698-699. DOl: 10.1038/205698a0.

[18] G. Sava, S. Pacor, G. Mestroni, E. Alessio, Clin. Exp. Metastasis 1992, 10, $273-$ 280. DOI: 10.1007/BF00133563.

[19] K. Lipponer, E. Vogel, B. K. Keppler, Metal-Based Drugs 1996, 3, 243-260. DOI: 10.1155/mbd.1996.243.

[20] W. Peti, T. Pieper, M. Sommer, B. K. Keppler, G. Giester, Eur. J. Inorg. Chem. 1999, 1999, 1551-1555. DOI: 10.1002/(SICI)1099-
0682(199909)1999:9\%3C1551::AID-EJIC1551\%3E3.0.CO;2-7.

[21] R. Trondl, P. Heffeter, M. A. Jakupec, W. Berger, B. K. Keppler, BMC Pharmacol. Toxicol. 2012, 13, A82. DOI: 10.1186/2050-6511-13-s1-a82.

[22] R. Trondl, P. Heffeter, C. R. Kowol, M. A. Jakupec, W. Berger, B. K. Keppler, Chem. Sci. 2014, 5, 2925-2932. DOI: 10.1039/C3SC53243G.

[23] R. E. Morris, R. E. Aird, P. del S. Murdoch, H. Chen, J. Cummings, N. D. Hughes, S. Parsons, A. Parkin, G. Boyd, D. I. Jodrell, P. J. Sadler, J. Med. Chem. 2001, 44, 3616-3621. DOI: 10.1021/jm010051m.

[24] P. J. Dyson, Chimia 2007, 61, 698-703. DOI: 10.2533/chimia.2007.698.

[25] T. S. Morais, A. Valente, A. I. Tomaz, F. Marques, M. H. Garcia, Fut. Med. Chem. 2016, 8, 527-544. D0I: 10.4155/fmc.16.7

[26] T. Morais, Síntese de novos complexos bioorganometálicos de ruténio e avaliação das suas propriedades anti-tumorais, Tese de Doutoramento em Química Inorgânica, Faculdade de Ciências da Universidade de Lisboa, 2013. repositorio.ul.pt/handle/10451/9908.

[27] L. Côrte-Real, B. Karas, P. Gírio, A. Moreno, F. Avecilla, F. Marques, B. T. Buckley, K. R. Cooper, C. Doherty, P. Falson, M. H. Garcia, A. Valente, Eur. J. Med. Chem. 2019, 163, 853-863. DOI: 10.1016/j.ejmech.2018.12.022.

[28] L. Côrte-Real, B. Karas, A. R. Brás, A. Pilon, F. Avecilla, F. Marques, A. Preto, B. T. Buckley, K. R. Cooper, C. Doherty, M. H. Garcia, A. Valente, Inorg. Chem 2019, 58, 9135-9149. DOI: 10.1021/acs.inorgchem.9b00735.

[29] A. Gandalovičová, D. Rosel, M. Fernandes, P. Veselý, P. Heneberg, V. Čermák, L. Petruželka, S. Kumar, V. Sanz-Moreno, J. Brábek, Trends Canc. 2017, 3 , 391-406. DOI: 10.1016/j.trecan.2017.04.008.

[30] P. Letrado, I. de Miguel, I. Lamberto, R. Díez-Martínez, J. Oyarzabal, Cancer Res. 2018, 78, 6048-6058. DOI: 10.1158/0008-5472.can-18-1029.

[31] B. F. Karas, L. Côrte-Real, C. L. Doherty, A. Valente, K. R. Cooper, B. T. Buckley, J. Appl. Toxicol. 2019, 39, 1173-1180. DOI: 10.1002/jat.3802.

\section{Leonor Côrte-Real}

Leonor Côrte-Real é investigadora de pós-doutoramento no grupo Bioinorganic Chemistry and Drug Development (BIOIN) do CQE - Ciências, Centro de Química Estrutural, onde desenvolve novos complexos metálicos com vista à terapia dirigida aos tumores. Possui um total de 16

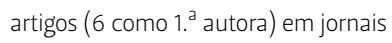
científicos com revisão por pares. Idcortereal@fc.ul.pt ORCID.org/0000-0002-4974-1075 